\title{
Parametrization of low-energy symmetric (e, 2e) differential cross section measurements*
}

\author{
A J Murray†, F H Read and N J Bowring \\ Department of Physics and Astronomy, Schuster Laboratory, Manchester University, Manchester
} M13 9PL, UK

Received 9 August 1996, in final form 24 September 1996

\begin{abstract}
Symmetric (e, 2e) experimental studies on helium over a very wide range of scattering geometries at energies from 1 to $50 \mathrm{eV}$ above the ionization threshold are parametrized in terms of a set of universal irreducible tensorial angular functions. Measurements from the Manchester and Paris experimental groups are used in this parametrization, which provides a complete picture of the symmetric ionization process. The use of the parametrization technique for deconvolution of the experimental angular resolution from measured results is discussed.
\end{abstract}

\section{Introduction}

A considerable body of experimental data now exists for (e, 2e) differential cross section (DCS) ionization studies of helium in which the scattered and ejected electrons are detected over a wide angular range with the same energy and at the same asymptotic scattering angles with respect to the incident electron direction (Murray and Read 1992, 1993 and references therein, Bowring et al 1996). Figure 1 shows the scattering geometry for these experiments. A detection plane spanned by the scattered and ejected electron momenta, $\boldsymbol{k}_{a}$ and $\boldsymbol{k}_{b}$, is defined. The incident electron momentum, $\boldsymbol{k}_{0}$, makes an angle, $\psi$, the 'gun angle', with respect to this plane, and the projection of this vector onto the detection plane defines the scattering angles, $\xi_{a}$ and $\xi_{b}$, of the two outgoing electrons. In all experiments carried out so far the condition, $\xi_{a}-\xi_{b}=\xi$, has been chosen, yielding a common normalization point at $\xi_{a}=\xi_{b}=\pi / 2$ for all gun angles, $\psi$.

The energy region from around 3 to $100 \mathrm{eV}$ excess energy is defined here as the 'intermediate energy' region. Studies at lower energies are considered to lie in the threshold region where correlation effects between outgoing electrons dominate the reaction, whereas DCS studies at energies in excess of $100 \mathrm{eV}$ are predominantly governed by single binary collisions between the incident and ejected electrons, the core playing a decreasing role in the reaction as this energy increases. This high-energy region is now successfully modelled to a large degree using the Born approximation either in second or higher order or by including distorted waves in the entrance and/or exit channels (for reviews see McCarthy and Weigold 1995, Coplan et al 1994).

The threshold region has been studied in detail using a number of different models ranging from the classical Wannier picture and its semi-classical and quantum mechanical derivatives (e.g Peterkop 1971, Rau 1971), through classical trajectory studies (Rost 1995) to

\footnotetext{
* http://es1.ph.man.ac.uk/AJM2/e2e/e2e1.htm
}

$\dagger$ E-mail address: A.Murray@fs3.ph.man.ac.uk 


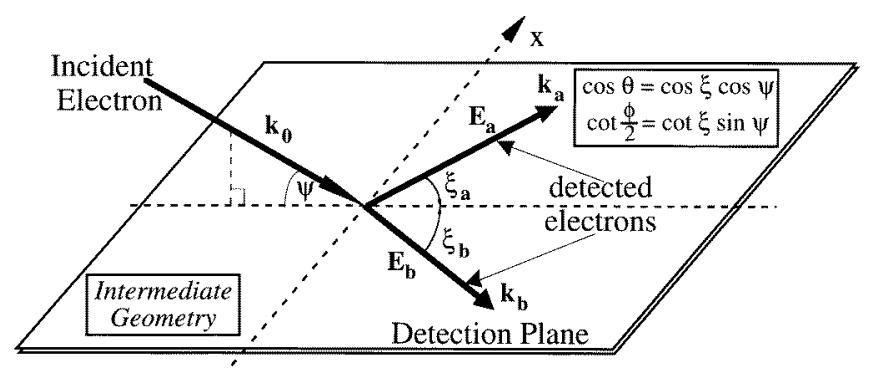

Figure 1. The intermediate experimental geometry. An electron with momentum $k_{0}$ incident at an angle $\psi$ on the plane spanned by the outgoing electrons ionizes a target helium atom. The scattered and ejected electrons of momenta $\boldsymbol{k}_{a}$ and $\boldsymbol{k}_{b}$ leave the interaction region at equal angles $\xi_{a}=\xi_{b}=\xi$ in this plane.

purely quantum mechanical treatments of ionization in this region (e.g. Altick 1985, Crothers 1986). Common to all these models is the importance of correlations between the outgoing electrons brought about through electrostatic coupling as they emerge from the reaction zone. As the excess energy above ionization decreases, the outgoing electrons have more time to mutually interact and so the probability that they will emerge asymptotically at a mutual angle of $\pi$ radians increases, whereas their 'memory' of the incident electron direction correspondingly decreases. The geometry chosen with $\xi_{a}=\xi_{b}=\xi$ thus increasingly constrains the DCS to a narrowing maximum at $\xi_{a}=\xi_{b}=\pi / 2$ as the excess energy decreases toward zero.

In the intermediate energy region the complexities of exchange, capture, incoming and outgoing channel distortions and short- and long-range correlations between all electrons and the core must be included, since all can play a significant role. Theoretical interest in this poorly understood region is increasing (e.g. Berekdar and Briggs 1994a, b) as more experimental data is accumulated, although at present no single theory adequately explains the data over all geometries.

In the present paper a model-independent parametrization of the ionization DCS is used. The parametrization is in terms of a complete orthogonal set of irreducible tensorial angular functions which define the correlation between any three vectors in space. In the present case, it is sensible to choose these vectors to be the unit ingoing electron momentum, $\hat{\boldsymbol{k}}_{0}$, and the unit outgoing electron moment, $\hat{\boldsymbol{k}}_{a}$ and $\hat{\boldsymbol{k}}_{b}$. This method of parametrization, first proposed for the (e, 2e) process by Klar and Fehr (1992), has a long and distinguished history in the field of nuclear particle interactions proceeding through sharply defined states, where the analysis directly gives information about the angular momenta carried into and out of the reaction (Biedenharn 1960, Brink and Satchler 1979, Biedenharn and Louke 1981). By contrast, in the $(\mathrm{e}, 2 \mathrm{e})$ reaction the intermediate state is not normally sharply defined, and so the parameters do not directly reveal the angular momenta associated with the reaction, but rather indicate the degree of correlation between ingoing and outgoing electrons.

This paper is divided into five main sections. Following this introduction the threshold (e, 2e) data of the Manchester and Paris groups described in the Wannier picture (HawleyJones et al 1992) is revisited to derive threshold data in the present $(\psi, \xi)$ geometry. The complete set of experimental symmetric data from 3 to $50 \mathrm{eV}$ excess energy is then graphically summarized in conventional form and these results are reviewed.

In the next section the new parametrization is introduced and the appropriate irreducible tensorial expressions derived. Choosing a symmetric geometry reduces the number of 
independent functions in the derivation and the appropriate selection rules for this case are given, together with examples of the functions used in the expansion. Two different methods are described for dealing with the experimentally inaccessible regions near $\xi=0^{\circ}$ and $180^{\circ}$. Some controversy exists for the absolute value of the DCS measured at different energies for the (e, 2e) process (Röder 1996, Murray et al 1992, Pochat et al 1993), and so equating the common point $\xi_{a}=\xi_{b}=\pi / 2$ to unity allows a simple renormalization to the correct absolute value once this discrepancy is resolved. Full three-dimensional DCS surfaces can thus be generated at all energies in this region from the parametrization.

Finally, it is shown that the model allows deconvolution of geometric effects on the signal due to the finite angular resolution of the spectrometer to be carried out by simply rescaling the parameters fitted to the measured experimental data.

\section{Parametrizing experimental data in the threshold region by the Wannier model}

In this section energy-independent fitting parameters in the Wannier model derived by Hawley-Jones et al (1992) are used to provide interpolated data between the coplanar results of Selles et al (1987a, b) and the perpendicular plane measurements of Hawley-Jones et al (1992). Briefly, the cross section is parametrized by Fournier-Lagarde et al (1984) as a coherent superposition of partial waves up to $L=2$ defining scattering in the reaction zone which is modulated by a Gaussian function that models the correlations between the outgoing electrons. The differential cross section, $\sigma$, is given by:

$$
\sigma=\left(\frac{1}{4}\left|f_{0}\right|^{2}+\frac{3}{4}\left|f_{1}\right|^{2}\right) \exp \left[\frac{-4 \gamma^{2} \ln 2}{\theta_{0}^{2} \sqrt{E_{\mathrm{exc}}}}\right]
$$

where $\gamma=\pi-\theta_{a b}$ and $\theta_{a b}=\cos ^{-1}\left[\cos \theta_{a} \cos \theta_{b}+\sin \theta_{a} \sin \theta_{b} \cos \left(\phi_{a}-\phi_{b}\right)\right]=\left(\xi_{a}+\xi_{b}\right)$ in the geometry chosen here. $E_{\text {exc }}$ is the excess energy shared between the outgoing electrons. For the symmetric geometry the triplet amplitude, $f_{1}$, is zero, leaving only the singlet partial wave contribution. As no absolute measurements are made the DCS is normalized to the $s$-wave amplitude, yielding singlet partial waves up to $L=2$ given by $f_{0}=f_{0}^{S}+f_{0}^{P}+f_{0}^{D}$ where

$$
\begin{aligned}
f_{0}^{S} & =E^{\alpha} \\
f_{0}^{P} & =E^{\alpha} P_{0}\left(\cos \theta_{a}+\cos \theta_{b}\right) \mathrm{e}^{\mathrm{i} \theta_{p_{0}}} \\
f_{0}^{P} & =E^{\alpha}\left[D_{0} \mathrm{e}^{\mathrm{i} \theta_{d_{0}}}+E^{-1 / 2} D_{0}^{\prime} \mathrm{e}^{\mathrm{i} \theta_{d_{0}^{\prime}}}\left(1+\frac{\gamma^{2}}{8}\right)\right]\left(\frac{3}{2}\left[\cos ^{2} \theta_{a}+\cos ^{2} \theta_{b}\right]-1\right) \\
& \quad-E^{\alpha}\left[D_{0} \mathrm{e}^{\mathrm{i} \theta_{d_{0}}}-E^{-1 / 2} D_{0}^{\prime} \mathrm{e}^{\mathrm{i} \theta_{d_{0}^{\prime}}}\left(1-\frac{\gamma^{2}}{8}\right)\right]\left(3 \cos \theta_{a} \cos \theta_{b}-\cos \theta_{a b}\right)
\end{aligned}
$$

and where $\alpha=0.1865$.

The energy-independent parameters in the above expressions calculated using the symmetric and asymmetric results of the Manchester and Paris groups yield:

Table 1.

\begin{tabular}{lllllll}
\hline$\theta_{0}(\mathrm{rad})$ & $P_{0}$ & $\theta_{p_{0}}(\mathrm{rad})$ & $D_{0}$ & $\theta_{d_{0}}(\mathrm{rad})$ & $D_{0}$ & $\theta_{d_{0}^{\prime}}(\mathrm{rad})$ \\
\hline $1.30 \pm 0.04$ & $1.6 \pm 0.3$ & $5.22_{-0.07}^{+0.04}$ & $0.84_{-0.04}^{+0.07}$ & $4.13_{-0.11}^{+0.04}$ & $2.13_{-0.28}^{+0.34}$ & $2.13_{-0.07}^{+0.05}$ \\
\hline
\end{tabular}




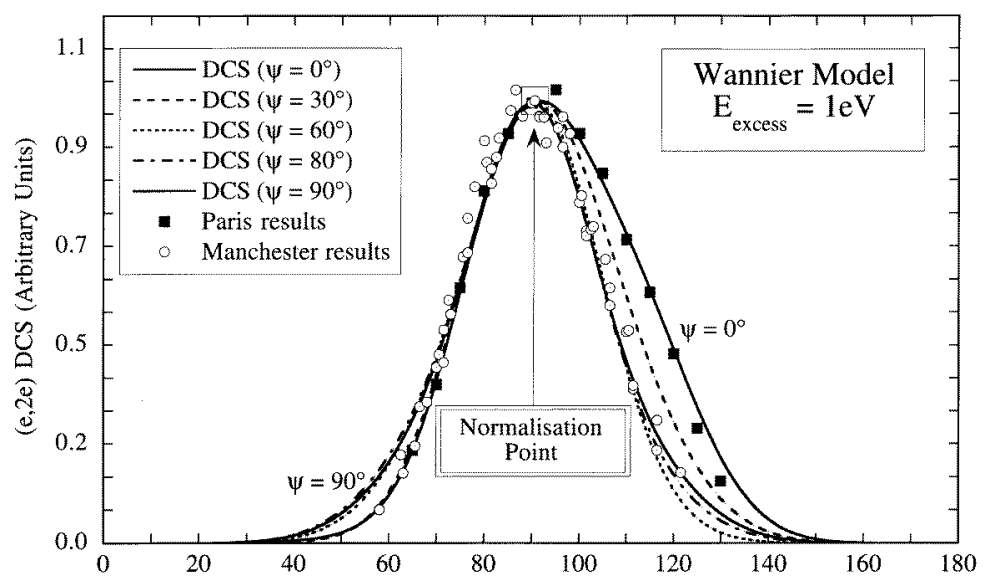

Figure 2. Normalized experimental results from the Paris and Manchester groups at $1 \mathrm{eV}$ above the ionization threshold of helium. The Paris coplanar and Manchester perpendicular plane results are parametrized in the Wannier model, the results of this parametrization being shown for various gun angles $\psi$.

The differential cross section is finally obtained in the $(\psi, \xi)$ geometry by applying the transformations:

$\cos \theta_{a}=\cos \theta_{b}=\cos \xi \cos \psi \quad \cot \frac{\phi}{2}=\cot \xi \sin \psi \quad$ where $\phi=\phi_{a}=\pi-\phi_{b}$.

Figure 2 shows the experimental results and the calculated DCS in the $(\psi, \xi)$ symmetric geometry at an excess energy, $E=1 \mathrm{eV}$, for incident electron beam angles, $\psi=0^{\circ}$, $30^{\circ}, 60^{\circ}, 80^{\circ}$ and $90^{\circ}$. The results have been renormalized to unity at the common point $\xi_{a}=\xi_{b}=\pi / 2$. The perpendicular plane results have an approximately Gaussian angular distribution, in contrast to the coplanar results which have a slight asymmetry in the backscatter direction, $(\xi>\pi / 2)$. There is no evidence at this energy of the complex structure expected from either a simple binary collision between scattered and ionized electrons into the forward direction, $(\xi \sim \pi / 4)$, or from the process in which a binary collision follows elastic scattering from the core of the incident electron into the backscatter region, $(\xi \sim 3 \pi / 4)$. Under the influence of strong outgoing electron correlations through electrostatic repulsion these scattering processes evolve into the single structure at $\xi=\pi / 2$, as observed.

\section{Experimental data in the intermediate energy region}

A comprehensive body of experimental data now exists for ionization of helium in the intermediate energy region over a very wide range of scattering geometries. These experiments have been carried out from the coplanar $\left(\psi=0^{\circ}\right)$ to the perpendicular plane geometry $(\psi=\pi / 2)$ for seven excess energies 3, 5, 10, 20, 30, 40 and $50 \mathrm{eV}$ above threshold (Murray and Read 1993, Bowring et al 1996). The complete symmetric data set is accessible electronically at the internet address http://es1.ph.man.ac.uk/AJM2/e2e/data/1-50eVdata.htm.

Figure 3 depicts these results and also shows the parametric fits as described in the following section. The DCS is assumed to be zero when $\xi=0$ and $\xi=\pi$ since the probability of detection of two electrons of the same energy in the same direction is very small. The regions between $\xi=0^{\circ}$ and $35^{\circ}$ and between $\xi=125^{\circ}$ and $180^{\circ}$ are 


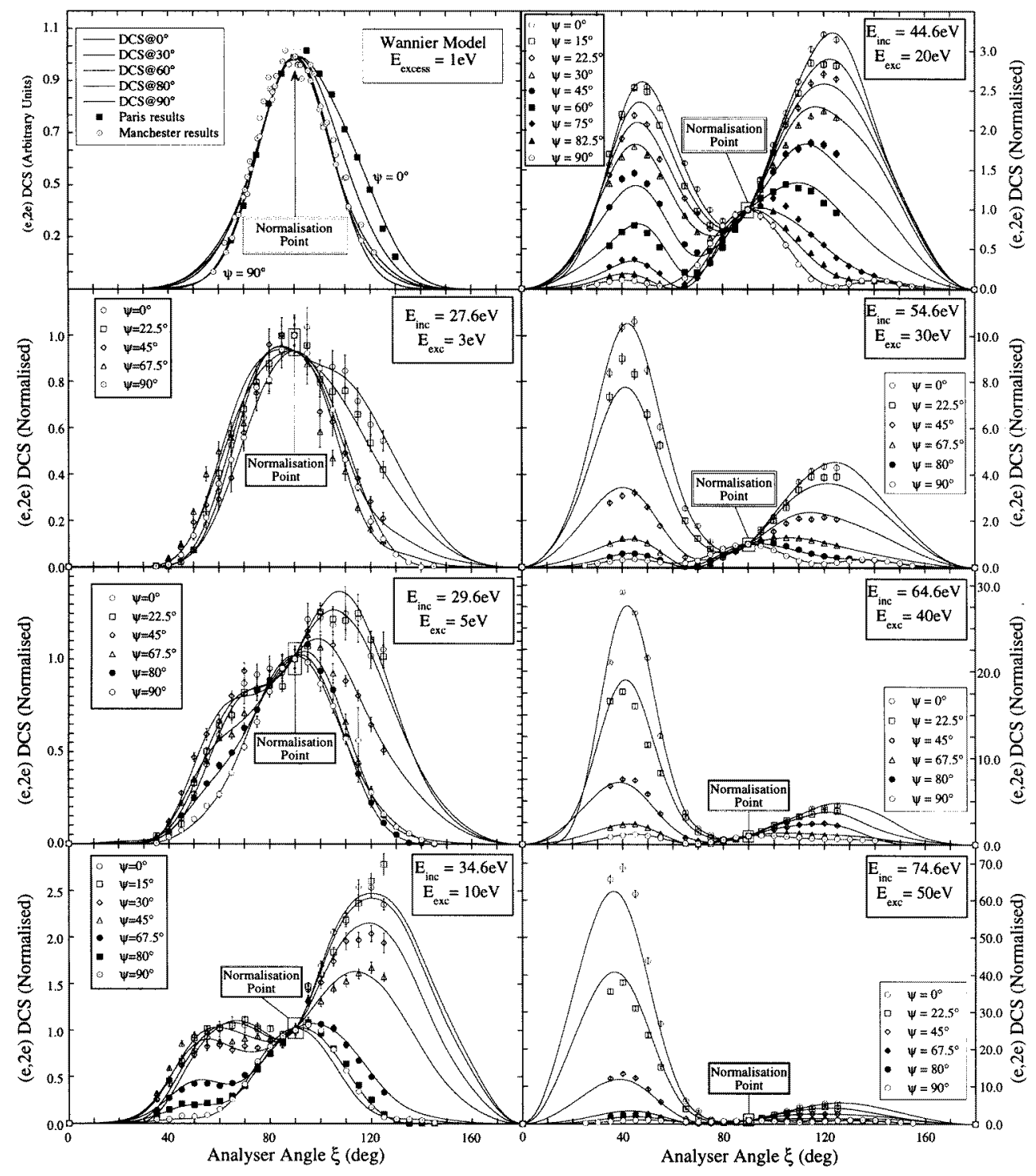

Figure 3. The complete set of experimental results from 1 to $50 \mathrm{eV}$ excess energy above the ionization energy of helium. The outgoing electrons share the excess energy equally, and are detected at equal scattering angles. The parametrization fitted to these results are shown.

experimentally inaccessible due to the physical size of the detectors and electron gun. In these regions the parametrization is constrained to be positive and monotonically decreasing towards zero at the end points.

At an excess energy of $1 \mathrm{eV}$ a single structure is observed due to strong outgoing electron correlation, as noted previously. At 3 and $5 \mathrm{eV}$ above the ionization threshold both a forward and a backward structure start evolving from the central peak at low-incident electron angles of $\psi$, whereas at higher angles of $\psi$ the DCS is still approximately Gaussian. Backward scattering, thought to be dominated by elastic scattering of the incoming electron from 
the atom into the backward direction of the detection plane followed by a binary collision with a valence electron, is the dominant contribution to the overall DCS structure up to an excess energy around $25 \mathrm{eV}$ where forward and backward peaks become approximately equal in magnitude (not shown). At higher excess energy $(30-50 \mathrm{eV})$ forward scattering starts to dominate, reflecting the importance of single binary collisions. The effect becomes increasingly important as the energy progresses into the high-energy regime.

It is noted that at the highest energy $E_{\text {exc }}=50 \mathrm{eV}$ the parametric fit to the low-angle coplanar results are not as satisfactory as for lower energies. This results from the problems of fitting the parametrization to the experimentally inaccessible regions in the gaps, where constraints are made to ensure that the fit has no points of inflection between the data and the end points. Unlike at lower energies, there are insufficient data points beyond the peak to accurately establish a well defined and appropriately constrained fit in this region, and so some uncertainty exists as to whether higher $l_{a}, l_{b}, l_{0}$ terms need to be added to improve the fit. Further work to establish data in these regions is necessary to resolve this discrepancy, however this requires significant modification to the experimental apparatus.

In the perpendicular plane $(\psi=\pi / 2)$ the single peak observed at low energies gradually evolves into a three-peak structure as the energy increases (the higher and lower peaks are simply reflections of each other). The relative magnitude of the central peak at $\xi=\pi / 2$ compared to the peaks at low and high values of $\xi$ decreases with increasing energy, until at $50 \mathrm{eV}$ excess energy the three peaks are of approximately the same magnitude (Murray et al 1992). Only the central peak is attainable through a single-collision process and there will additionally be significant contributions to this peak through outgoing electron correlations at the lower energies. The non-central peaks can only arise through higher-order collision processes.

The DCS has certain symmetries defined by the experimental constraints. Indistinguishability of the outgoing electrons requires the DCS at $(\psi, \xi)$ to be equated to that at $(\psi, 2 m \pi-\xi)$ where $m$ is an integer. Reflection symmetry is necessary in the detection plane since no preferred direction is defined with respect to this plane, and hence $\sigma(\psi, \xi)=\sigma(-\psi, \xi)$. As the gun angle reaches $\pi / 2$ the projection of $\hat{\boldsymbol{k}}_{0}$ onto the detection plane becomes undefined and so only the angle $\left(\xi_{a}+\xi_{b}\right)$ is relevant, as a consequence of which $\sigma(\pi-\psi, \xi)=\sigma(\psi, \pi-\xi)$. All these symmetries are invoked when parametrizing the experimental data.

\section{Parametrizing the experimental data}

In this section the parametrization of the data is described, including details of the fitting procedures. The experimental symmetries outlined in the previous section allow additional data to be inferred in the region $\pi / 2 \geqslant \psi \geqslant 2 \pi$. In the experimentally inaccessible regions centred as $\xi=0^{\circ}$ and $\xi=\pi$ the DCS is constrained to be positive and to have no points of inflection, and at the angles $\xi=0^{\circ}$ and $\pi$ themselves the DCS is put equal to zero.

\subsection{General model}

When electron spin is not considered the (e, 2e) differential cross section is a function only of the momenta of the ingoing and outgoing electrons. Hence

$$
\sigma=\sigma\left(\boldsymbol{k}_{0}, \boldsymbol{k}_{a}, \boldsymbol{k}_{b}\right)
$$


It can be shown (Biedenharn 1960) that a complete orthogonal basis upon which to expand the angular dependence of the cross section is given by the functions

$$
\begin{aligned}
& I_{l_{a} l_{b} l_{0}}\left(\hat{\boldsymbol{k}}_{a}, \hat{\boldsymbol{k}}_{b}, \hat{\boldsymbol{k}}_{0}\right) \\
& \quad=\frac{(-1)^{l_{b}-l_{a}} 4 \pi^{3 / 2}}{\sqrt{\left(2 l_{a}+1\right)\left(2 l_{b}+1\right)}} \sum_{m_{a} m_{b}}\left(\begin{array}{ccc}
l_{a} & l_{b} & l_{0} \\
m_{a} & m_{b} & -m_{0}
\end{array}\right) Y_{l_{a} m_{a}}\left(\hat{\boldsymbol{k}}_{a}\right) Y_{l_{b} m_{b}}\left(\hat{\boldsymbol{k}}_{b}\right) Y_{l_{0} m_{0}}^{*}\left(\hat{\boldsymbol{k}}_{0}\right)
\end{aligned}
$$

where $Y_{l m}(\hat{\boldsymbol{k}})$ is a spherical harmonic. Expanding the DCS in terms of this basis then gives

$$
\sigma\left(\boldsymbol{k}_{a}, \boldsymbol{k}_{b}, \boldsymbol{k}_{0}\right)=\sum_{l_{a} l_{b} l_{0}} B_{l_{a} l_{b} l_{0}} I_{l_{a} l_{b} l_{0}}\left(\hat{\boldsymbol{k}}_{a}, \hat{\boldsymbol{k}}_{b}, \hat{\boldsymbol{k}}_{0}\right)
$$

where $B_{l_{a} l_{b} l_{0}}$ define all non-angular dependent terms characterizing the reaction. In the present study these are functions only of the excess energy.

The choice of angular functions, $I_{l_{a} l_{b} l_{0}}$, required to characterize a general (e, 2e) DCS is restricted by the symmetries inherent in the ionization process. From the phase of the spherical harmonics it is clear that

$$
I_{l_{a} l_{b} l_{0}}\left(-\hat{\boldsymbol{k}}_{a},-\hat{\boldsymbol{k}}_{b},-\hat{\boldsymbol{k}}_{0}\right)=(-1)^{l_{a}+l_{b}+l_{0}} I_{l_{a} l_{b} l_{0}}\left(\hat{\boldsymbol{k}}_{a}, \hat{\boldsymbol{k}}_{b}, \hat{\boldsymbol{k}}_{0}\right) .
$$

Reflection symmetry requires, however, that

$$
I_{l_{a} l_{b} l_{0}}\left(-\hat{\boldsymbol{k}}_{a},-\hat{\boldsymbol{k}}_{b},-\hat{\boldsymbol{k}}_{0}\right)=I_{l_{a} l_{b} l_{0}}\left(\hat{\boldsymbol{k}}_{a}, \hat{\boldsymbol{k}}_{b}, \hat{\boldsymbol{k}}_{0}\right) .
$$

Hence $\left(l_{a}+l_{b}+l_{0}\right)$ must be an even integer. The presence of the Wigner $3 j$ term in equation (5) requires that $\left(l_{a}, l_{b}, l_{0}\right)$ should satisfy the triangular condition. The DCS must also be invariant upon exchange of electrons $a$ and $b$. Since the $3 j$ term requires that

$$
I_{l_{b} l_{a} l_{0}}\left(\hat{\boldsymbol{k}}_{b}, \hat{\boldsymbol{k}}_{a}, \hat{\boldsymbol{k}}_{0}\right)=(-1)^{l_{a}+l_{b}+l_{0}} I_{l_{a} l_{b} l_{0}}\left(\hat{\boldsymbol{k}}_{a}, \hat{\boldsymbol{k}}_{b}, \hat{\boldsymbol{k}}_{0}\right)
$$

it follows that

$$
B_{l_{b} l_{a} l_{0}}\left(\left|\boldsymbol{k}_{b}\right|,\left|\boldsymbol{k}_{a}\right|\right)=B_{l_{a} b_{b} l_{0}}\left(\left|\boldsymbol{k}_{a}\right|,\left|\boldsymbol{k}_{b}\right|\right) .
$$

Hence the summation in equation (6) can be restricted to $l_{b} \geqslant l_{a}$.

For the present experiments it is sensible to define the $z$-axis along $\boldsymbol{k}_{0}$ and to let the $x$-axis lie in the detection plane spanned by $\hat{\boldsymbol{k}}_{a}$ and $\hat{\boldsymbol{k}}_{b}$. The polar angle of the vectors $\hat{\boldsymbol{k}}_{a}$ and $\hat{\boldsymbol{k}}_{b}$ are then defined as $\left(\theta_{a}, \phi_{a}\right)$ and $\left(\theta_{b}, \phi_{b}\right)$. The angular functions then have the form

$$
\begin{gathered}
I_{l_{a} l_{b} l_{0}}\left(\theta_{a}, \phi_{a}, \theta_{b}, \phi_{b}\right)=(-1)^{l_{b}-l_{a}} \sqrt{\left(2 l_{0}+1\right)} \sum_{m}\left(\begin{array}{ccc}
l_{a} & l_{b} & l_{0} \\
m & -m & 0
\end{array}\right)\left[\frac{\left(l_{a}-|m|\right) !\left(l_{b}-|m|\right) !}{\left(l_{a}+|m|\right) !\left(l_{b}+|m|\right) !}\right]^{1 / 2} \\
\times P_{l_{a}}^{|m|}\left(\cos \theta_{a}\right) P_{l_{b}}^{|m|}\left(\cos \theta_{b}\right) \mathrm{e}^{\mathrm{i} m\left(\phi_{a}-\phi_{b}\right)} .
\end{gathered}
$$

In general the complete set of angular functions is required to fully characterize the differential cross section, but with the present geometry the relationships $\theta_{b}=\theta_{a}$ and $\phi_{b}=\pi-\phi_{a}$ cause many of the angular functions to be linearly related. Thus for certain values of $l_{a}, l_{b}$ and $l_{0}$ we can write:

$$
I_{l_{a} l_{b} l_{0}}(\theta, \phi)=\sum_{l_{a}^{\prime} b_{b}^{\prime} l_{0}^{\prime}} c_{l_{a}^{\prime} l_{b}^{\prime} l_{0}^{\prime}} I_{l_{a}^{\prime} l_{b}^{\prime} l_{0}^{\prime}}(\theta, \phi)
$$

and only a subset of angular functions is required. The choice of $l_{a}, l_{b}$ and $l_{0}$ used to define the subset is arbitrary within the constraint that the angular functions of the subset must be unrelated. It is convenient to choose the set for which $l_{a}=l_{b}$ when $l_{0}$ is even and $\left(l_{a}, l_{0}\right)<l_{b}$ when $l_{0}$ is odd, in contrast to the set chosen in Murray et al (1994). All other angular functions can be related to this set using equation (12). 


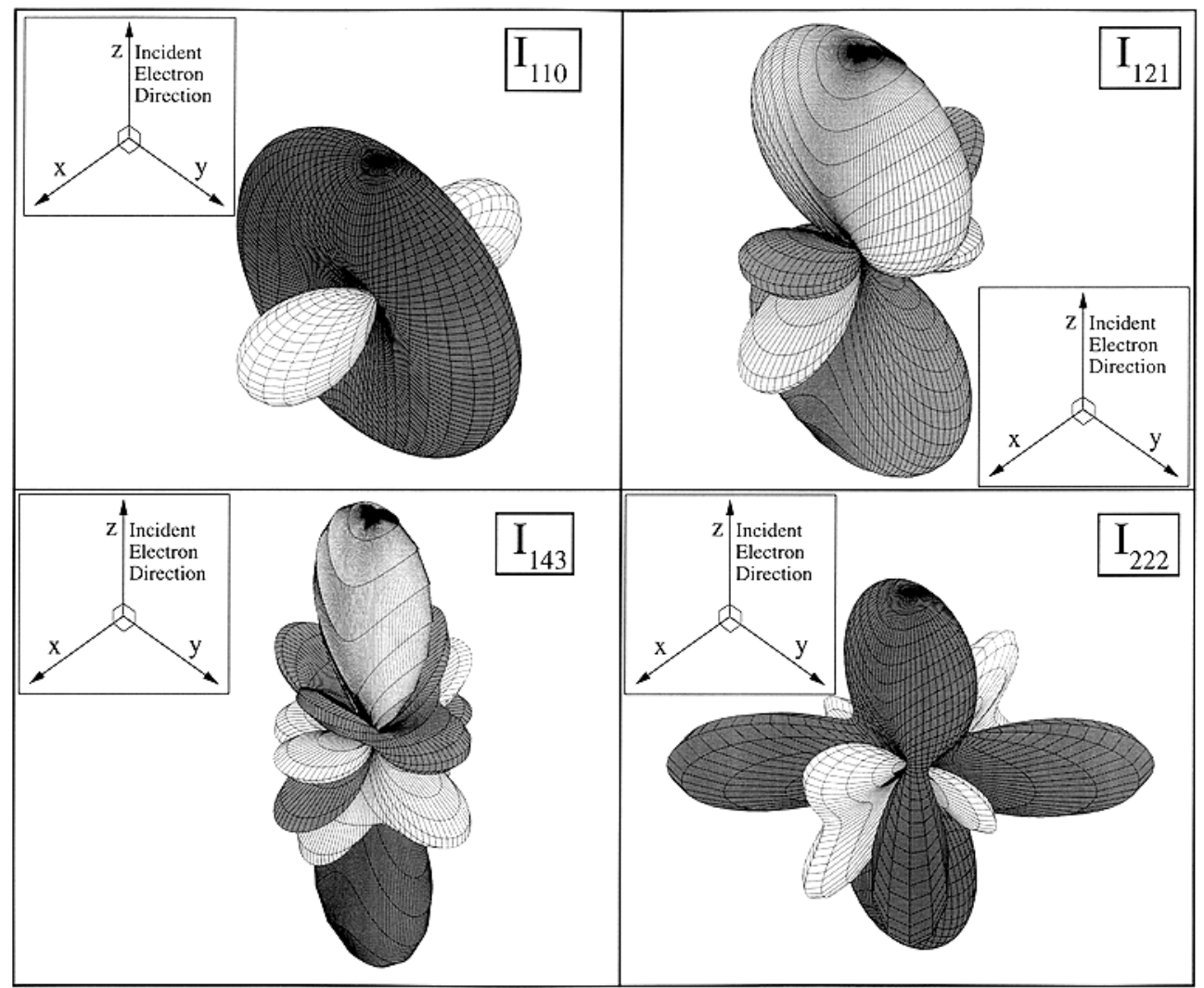

Figure 4. Examples of the angular functions which are used in the expansion of the coupled equations given in equation (13). The incident electron direction is defined as the $z$-axis as shown. The functions take on positive and negative values as the polar angle is varied throughout space, the lighter shading representing the function when positive and the darker shading representing the function when negative.

As an example, the function $I_{134}$ and $I_{235}$ can be written as a summation of six functions:

$$
\begin{aligned}
& I_{134}=\frac{-5}{9 \sqrt{7}} I_{000}-\frac{11}{6 \sqrt{21}} I_{110}-\frac{5 \sqrt{2}}{12 \sqrt{21}} I_{112}-\frac{\sqrt{5}}{18 \sqrt{7}} I_{220}-\frac{5 \sqrt{2}}{36} I_{222}+\frac{\sqrt{10}}{3} I_{224} \\
& I_{235}=\frac{\sqrt{5}}{\sqrt{4} 2} I_{011}+\frac{2 \sqrt{6}}{\sqrt{35}} I_{033}+\frac{\sqrt{10}}{\sqrt{21}} I_{055}+\frac{4}{\sqrt{21}} I_{121}+\frac{3 \sqrt{6}}{\sqrt{35}} I_{143}+\frac{\sqrt{6}}{6} I_{231} .
\end{aligned}
$$

Figure 4 shows three-dimensional representations of the basis functions used in these expansions. It should be noted that, although the basis functions increase in complexity as the values of $l_{a}, l_{b}$ and $l_{0}$ increase, their weighted summation (equation (6)) may still have a simple form.

\subsection{Fitting procedures}

The subset of angular functions, $I_{l_{a} l_{b} l_{0}}$, with $\left(l_{a}, l_{b}, l_{0}\right) \leqslant 15$ was generated from equation (11) using the analytic programming package Maple $\mathrm{V}$, which also produced FORTRAN codes for these functions. Analytic derivation was preferred over numerical 
generation since this was inherently more accurate and could be computed with significantly greater speed. The analytic expressions were checked using a numerical derivation of $I_{l_{a} l_{b} l_{0}}$ at specific angles $\left(\theta_{a}, \phi_{a}, \theta_{b}, \phi_{b}\right)$ to establish that no errors occurred in their generation.

Two least squares fitting procedures were used to parametrize the experimental data, each dealing with the inaccessible experimental regions in different ways. The quantity to be minimized is the $\chi^{2}$ function

$$
\chi^{2}=\sum_{i} w_{i}\left[\sigma_{\text {expt }}\left(\boldsymbol{k}_{a}, \boldsymbol{k}_{b}, \boldsymbol{k}_{0}\right)-\sum_{l_{a} l_{b} l_{0}} B_{l_{a} l_{b} l_{0}}\left(E_{\text {exc }}\right) I_{l_{a} l_{b} l_{0}}\left(\hat{\boldsymbol{k}}_{a}, \hat{\boldsymbol{k}}_{b}, \hat{\boldsymbol{k}}_{0}\right)\right]^{2}
$$

where the summation is over the experimental data points, $i$, each with an associated weight, $w_{i}$, evaluated from the uncertainty in the data.

In the first procedure a linear least squares fit was used to obtain the unknown $B_{l_{a} l_{b} l_{0}}$. To facilitate the requirement that the fitting function should be positive and monotonic in the experimentally inaccessible regions, extra pseudo-points were added to the fitted data in these regions. These were produced from a spline fit through the data which was constrained to pass through zero at $\xi=-\pi / 2,0^{\circ},+\pi / 2$ and $+\pi$. Experimental data in the regions $-\pi / 2 \leqslant \xi \leqslant 0^{\circ}$ and $+\pi / 2 \leqslant \xi \leqslant+\pi$ where generated using the symmetries defined in section 3 . The artificially generated points were given low weighting to minimize the constraints placed upon the fit. This linear fitting was fast and reliable, and yielded excellent starting parameters for the second fitting method.

In the second procedure the necessary assumptions in the inaccessible regions were satisfied without inclusion of artificial pseudo-points. A Simplex method (Nelder and Mead 1965) was used to minimize the $\chi^{2}$ function by adjusting the $B_{l_{a} l_{b} l_{0}}$ parameters. Any trial for which the fitting function did not obey the assumptions was rejected and the Simplex routine was reset to explore alternative regions of the $\chi^{2}$ surface. This method converged far more slowly than the linear fitting method which required only a single matrix inversion, but was preferred since it did not require artificial data generation.

To establish the maximum significant values of $l_{a}, l_{b}$ and $l_{0}$ a systematic study was carried out on the normalized $\chi^{2}$ as a function of increasing numbers of angular functions used in the fitting procedure. The statistical $F$-test (Bevington and Robinson 1990) was used to establish the validity of further terms in the fit. This test uses the ratio of the normalized $\chi^{2}$ as the number of terms is increased and compares this with the expected variation for a statistical fit. Consideration of the data sets at all excess energies established that 44 angular functions up to $l_{a}, l_{b}=7$ and $l_{0}=6$ gave the best overall fit to the data for all energies. These functions were then used as a basis for fitting all the data so that a comparison could be made between different data sets at different energies.

To estimate the uncertainties in the $B_{l_{a} l_{b} l_{0}}$ parameters using the Simplex technique the experimental data points were given random variations based upon a Normal distribution. The program was rerun many times using these random variations to estimate the uncertainties in the fitting parameters.

Figure 5 shows the 44 parameters for the eight data sets normalized to unity at $\xi=\pi / 2$, including the fit to the Wannier model at $1 \mathrm{eV}$. The uncertainty in each parameter is shown as a variation of the length of the corresponding column.

There is a clear difference between the parameters in the threshold region compared to those in the higher-energy region. At an excess energy of $1 \mathrm{eV}$ only $l_{0}=0-4$ contribute, and the $l_{a}$ and $l_{b}$ terms are all of the same sign and therefore reinforce each other in each $l_{0}$ manifold. The limitation on $l_{0 \max }$ reflects the bilinear fit to the $L=0,1,2$ partial wave coherent summation in the Wannier model, whereas the equal signs of the $l_{a}$ and $l_{b}$ terms reflect the simplicity of the lobe structure. The relative magnitudes of the terms of each 


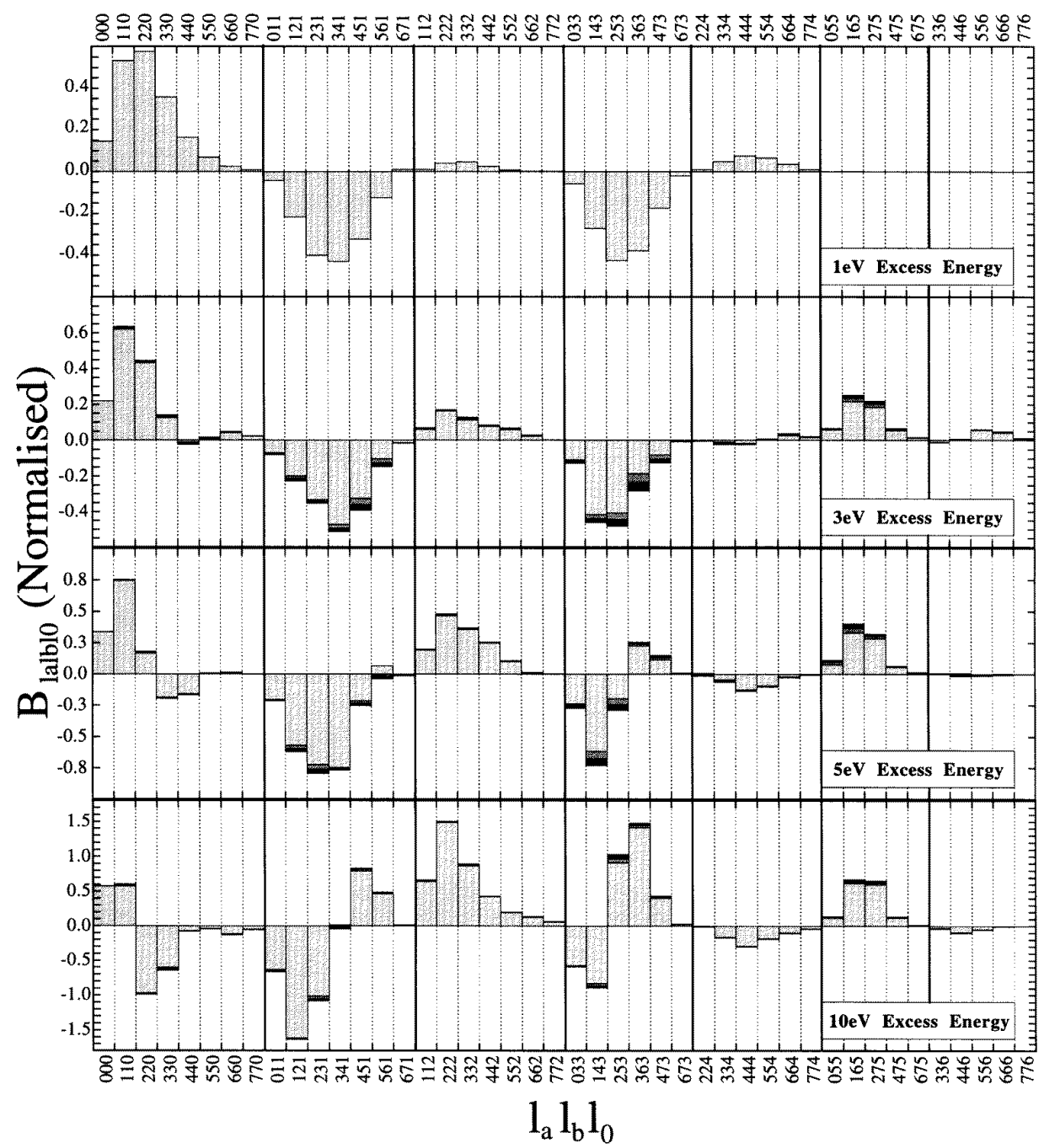

(a)

Figure 5. (a) The fitted parameters $B_{l_{a} l_{b} l_{0}}$ with their associated errors shown as variations in each column for the experimental results from 1 to $10 \mathrm{eV}$ excess energy. The experimental results have been normalized to unity at the common point $\xi=\pi / 2$. (b) The fitted parameters $B_{l_{a} l_{b} l_{0}}$ with their associated errors shown as variations in each column for the experimental results from 20 to $50 \mathrm{eV}$ excess energy. The experimental results have been normalized to unity at the common point $\xi=\pi / 2$.

manifold combine to produce the lobe structure at the mutual angle of $\pi$ radians.

As the energy increases this regular pattern quickly disappears as the forward and backward lobes evolve from the central 'Gaussian' profile. Higher-order $l_{0}$ correlation terms become significant as the intermediate energy region is entered, indicating an increasing contribution of higher-order partial waves to the DCS. Additionally the $l_{a}$ and $l_{b}$ terms start to compete in magnitude and sign to produce the more complex structures observed. At excess energies above about $30 \mathrm{eV}$ the parameters tend to stabilize in sign and vary smoothly with energy. The maximum significant number of $l_{a}$ and $l_{b}$ terms in each $l_{0}$ manifold also 


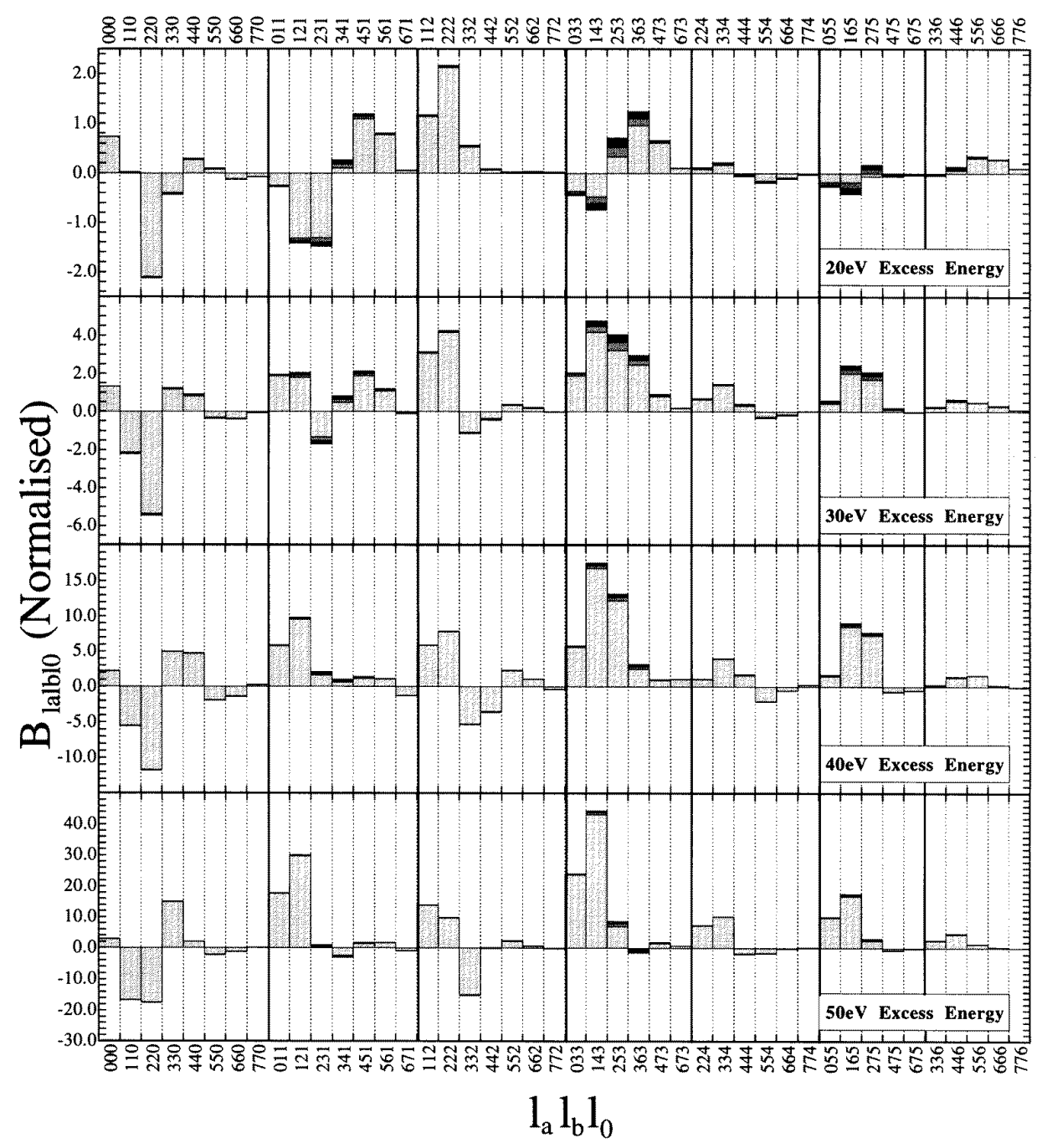

(b)

Figure 5. (Continued)

decreases as the energy increases, indicating that the number of partial waves required in the outgoing channel decreases with increasing energy.

\subsection{Parametrizing the DCS in terms of excess energy}

It is useful to establish a technique for interpolating between the results at the eight discrete excess energies, to enable the DCS to be estimated as a function of the three angular and one energy variables. Because the DCS are normalized to unity at the common point $\xi=\pi / 2$, only the relative changes as a function of energy can be determined, leaving the true energy dependence to be established using a single scaling function once absolute DCS values at the common normalization point have been measured.

As no physical model yet exists, a cubic spline has been interpolated through the eight 
$B_{l_{a} l_{b} l_{0}}$ data sets as a function of the ratio of excess energy to ionization energy. A ninth-order polynomial fit to this spline curve then yields a compact set of parameters, $\beta_{l_{a} l_{b} l_{0}, j}$, which closely emulate the cubic spline through the data. A total of $440 \beta$-parameters then define the normalized DCS as a function of energy and angle. These parameters, together with the associated $I_{l_{a} l_{b} l_{0}}$ angular functions are accessible electronically at the internet address http://es1.ph.man.ac.uk/AJM2/e2e/param/param.htm.

The $\beta$-parameters allow normalized DCS surfaces to be generated at all energies from 1 to $50 \mathrm{eV}$ for all angles $(\psi, \xi)$. A complete set of three-dimensional images of these surfaces has been generated, allowing the evolution of the DCS with energy to be observed. These images, together with a viewable Quicktime file can also be accessed at the above internet address.

\section{Application of the model to the deconvolution of experimental angular widths}

Comparisons between theoretical and experimental differential cross sections always contain uncertainties because the experimental signal is averaged over the finite energy and angular spreads introduced by the electron gun, the electron analysers and the finite size of the interaction region.

We now show that parametrization in terms of the angular functions, $I_{l_{a} l_{b} l_{0}}$, allows a considerable degree of simplification to be made, since integration over the incident electron pencil angle and the pencil angles of the detectors is to a good approximation equivalent to a simple rescaling of the $B_{l_{a} l_{b} l_{0}}$ parameters. Alternatively, theoretical results defining the DCS can be expanded into this set of angular functions and then rescaled in a similar way.

Consider figure 6: The pencil angle, $\gamma_{0}$, of the incident electron beam defines a cone centred about the $z$-axis (assuming zero beam angle), the incident electron direction, $\hat{\boldsymbol{k}}_{0}$, being confined to lie within this cone. The detectors view the interaction volume as shown. The vectors, $\boldsymbol{r}_{a, b}^{\prime}$, from an ionization event to the centre of the detector pencil angles (defined electrostatically by the apertures in the input lenses) are related to the vectors, $\boldsymbol{r}_{a, b}$, from the centre of the interaction region since $\boldsymbol{r}_{a, b}^{\prime}=\boldsymbol{r}_{a, b}-\boldsymbol{r}_{\text {int }}$. To a good approximation for small solid angles and a small interaction volume the pencil angles centred around $\boldsymbol{r}_{a, b}^{\prime}$ can be considered symmetrically centred around $\boldsymbol{r}_{a, b}$ with pencil angles of $\gamma_{a, b}$. The detected electron directions, $\hat{\boldsymbol{k}}_{a}$ and $\hat{\boldsymbol{k}}_{b}$, then are constrained to lie within these conical surfaces.

The experimental signal at any given $\left(\theta_{a}, \phi_{a}, \theta_{b}, \phi_{b}\right)$ represents a summation over all possible incident-beam directions within the incident-beam cone together with all possible events within the detection cones, summed over the distribution of the ionization events in the interaction volume. Hence the experimental signal $I_{\text {expt }}\left(\theta_{a}, \phi_{a}, \theta_{b}, \phi_{b}\right)$ is given by:

$$
\begin{aligned}
& I_{\text {expt }}\left(\theta_{a}, \phi_{a}, \theta_{b}, \phi_{b}\right) \sim \frac{1}{V} \sum_{\boldsymbol{r}} \rho\left(\boldsymbol{r}_{\text {int }}\right) \sum_{\boldsymbol{k}_{0}^{\gamma 0}} \sum_{\boldsymbol{k}_{a}^{\gamma a}} \sum_{\boldsymbol{k}_{b}^{\gamma b}} \sigma\left(\boldsymbol{k}_{a}, \boldsymbol{k}_{b}, \boldsymbol{k}_{0}\right) \\
& \rightarrow \frac{\int_{r_{\text {int }}} \rho\left(\boldsymbol{r}_{\text {int }}\right) \int_{\Omega_{k_{0}}} \int_{\Omega_{k_{a}}} \int_{\Omega_{k_{b}}} \sigma\left(\boldsymbol{k}_{a}, \boldsymbol{k}_{b}, \boldsymbol{k}_{0}\right) \mathrm{d} \Omega_{\boldsymbol{k}_{a}} \mathrm{~d} \Omega_{\boldsymbol{k}_{b}} \mathrm{~d} \Omega_{\boldsymbol{k}_{0}} \mathrm{~d} \boldsymbol{r}_{\text {int }}}{\int_{\boldsymbol{r}_{\text {int }}} \int_{\Omega_{k_{0}}} \int_{\Omega_{k_{a}}} \int_{\Omega_{k_{b}}} \mathrm{~d} \Omega_{k_{0}} \mathrm{~d} \Omega_{\boldsymbol{k}_{a}} \mathrm{~d} \Omega_{\boldsymbol{k}_{b}} \mathrm{~d} \boldsymbol{r}_{\text {int }}}
\end{aligned}
$$

where $\rho\left(\boldsymbol{r}_{\text {int }}\right)$ defines the density profile of ionization events within the interaction volume (Buckman et al 1993) and the integrals in the denominator normalize the signal.

Integration over the incident and detected electron cone angles is simplified by the 
expansion of the DCS into the angular functions:

$$
\begin{aligned}
\iiint \sigma\left(\boldsymbol{k}_{a}, \boldsymbol{k}_{b}, \boldsymbol{k}_{0}\right) \mathrm{d} \Omega_{\boldsymbol{k}_{a}} \mathrm{~d} \Omega_{\boldsymbol{k}_{b}} \mathrm{~d} \Omega_{\boldsymbol{k}_{0}} \\
=\sum_{l_{a} l_{b} l_{0}} \frac{(-1)^{l_{b}-l_{a}} 4 \pi^{3 / 2}}{\sqrt{\left(2 l_{a}+1\right)\left(2 l_{b}+1\right)}} \sum_{m_{a} m_{b}}\left(\begin{array}{ccc}
l_{a} & l_{b} & l_{0} \\
m_{a} & m_{b} & -m_{0}
\end{array}\right) \\
\quad \times \int_{\Omega_{k_{0}}} Y_{l_{0} m_{0}}^{*}\left(\hat{\boldsymbol{k}}_{0}\right) \int_{\Omega_{k_{a}}} Y_{l_{a} m_{a}}\left(\hat{\boldsymbol{k}}_{a}\right) \mathrm{d} \Omega_{a} \int_{\Omega_{k_{b}}} Y_{l_{b} m_{b}}\left(\hat{\boldsymbol{k}}_{b}\right) \mathrm{d} \Omega_{b} \mathrm{~d} \Omega_{0} .
\end{aligned}
$$

Consider the integral over detector $a$ in this expansion. Integration over the solid angle, $\Omega_{k a}$, is simplified by rotating the coordinate system $(x, y, z)$ into the new coordinate system $\left(x_{a}, y_{a}, z_{a}\right)$ as shown in figure 8 where the $z_{a}$-axis is in the direction of $\boldsymbol{r}_{a}^{\prime}$, since in this coordinate system

$$
\int \mathrm{d} \Omega_{a} \rightarrow \int_{\beta_{a}=0}^{\gamma_{a}} \int_{\varepsilon_{a}=0}^{2 \pi} \sin \beta_{a} \mathrm{~d} \beta_{a} \mathrm{~d} \varepsilon_{a} .
$$

As the initial coordinates in this rotation are located around the point $\left(\boldsymbol{r}_{\text {int }}, \theta_{\mathrm{int}}, \phi_{\text {int }}\right)$ in the interaction volume it is here that any error due to integration over asymmetry of the detection cone occurs. For the solid angles used in these experiments this error is less than 1\%; however, for much larger solid angles of detection, or for extended interaction volumes, a more exact calculation would be required. It should be noted that applying this assumption decouples the integration over the gun and detector pencil angles from integration over $\boldsymbol{r}=\left(r_{\text {int }}, \theta_{\text {int }}, \phi_{\text {int }}\right)$ in equation (15), and hence the variation of experimental signal with respect to the density profile of ionization events $\rho\left(\boldsymbol{r}_{\text {int }}\right)$ can be written as a constant multiplier outside the integrations given in equation (16).

The spherical harmonics transform under this rotation according to the unitary rotation matrix $D_{m m^{\prime}}^{l}(\omega)$ (Rose 1953, Edmonds 1957):

$$
\begin{gathered}
Y_{l_{a} m_{a}}\left(\hat{\boldsymbol{k}}_{a}\right)=Y_{l_{a} m_{a}}\left(\theta_{a}, \phi_{a}\right)=\sum_{m^{\prime}=-l_{a}}^{m^{\prime}=l_{a}} Y_{l_{a} m^{\prime}}\left(\hat{\boldsymbol{k}}_{a}^{\prime}\right) D_{m_{a} m^{\prime}}^{l_{a}}(\alpha, \beta, \gamma) \\
=\sum_{m^{\prime}=-l_{a}}^{m^{\prime}=l_{a}} Y_{l_{a} m^{\prime}}\left(\beta_{a}, \varepsilon_{a}\right) D_{m_{a} m^{\prime}}^{l_{a}}(\alpha, \beta, \gamma)
\end{gathered}
$$

where $(\alpha, \beta, \gamma)$ are Euler angles that specify the rotation, which in the present case are $\left(\phi_{a}, \theta_{a}, 0\right)$. The integral over $\Omega_{a}$ therefore becomes:

$$
\int Y_{l_{a} m_{a}}\left(\hat{\boldsymbol{k}}_{a}\right) \mathrm{d} \Omega_{a}=\sum_{m^{\prime}=-l_{a}}^{m^{\prime}=l_{a}} D_{m_{a} m^{\prime}}^{l_{a}}(\alpha, \beta, \gamma) \int_{\beta_{a}=0}^{\gamma_{a}} \int_{\varepsilon_{a}=0}^{2 \pi} Y_{l_{a} m^{\prime}}\left(\beta_{a}, \varepsilon_{a}\right) \sin \beta_{a} \mathrm{~d} \beta_{a} \mathrm{~d} \varepsilon_{a} .
$$

By using the recursive forms of the Legendre polynomials this integral becomes

$$
\int Y_{l_{a} m_{a}}\left(\hat{\boldsymbol{k}}_{a}\right) \mathrm{d} \Omega_{a}=Y_{l_{a} m_{a}}\left(\theta_{a}, \phi_{a}\right) \frac{\cos \gamma_{a} P_{l_{a}}\left(\cos \gamma_{a}\right)-P_{l_{a}-1}\left(\cos \gamma_{a}\right)}{\left(l_{a}+1\right)} .
$$

The normalization integral in equation (15) simply reduces to

$$
\int_{\Omega_{k_{a}}} \mathrm{~d} \Omega_{k_{a}}=\cos \left(\gamma_{a}\right)-1
$$

and hence the spherical harmonic, $Y_{l_{a} m_{a}}$, is attenuated by the factor:

$$
A_{l_{a}}\left(\gamma_{a}\right)=\frac{\cos \gamma_{a} P_{l_{a}}\left(\cos \gamma_{a}\right)-P_{l_{a}-1}\left(\cos \gamma_{a}\right)}{\left(l_{a}+1\right)\left(\cos \gamma_{a}-1\right)} \text {. }
$$




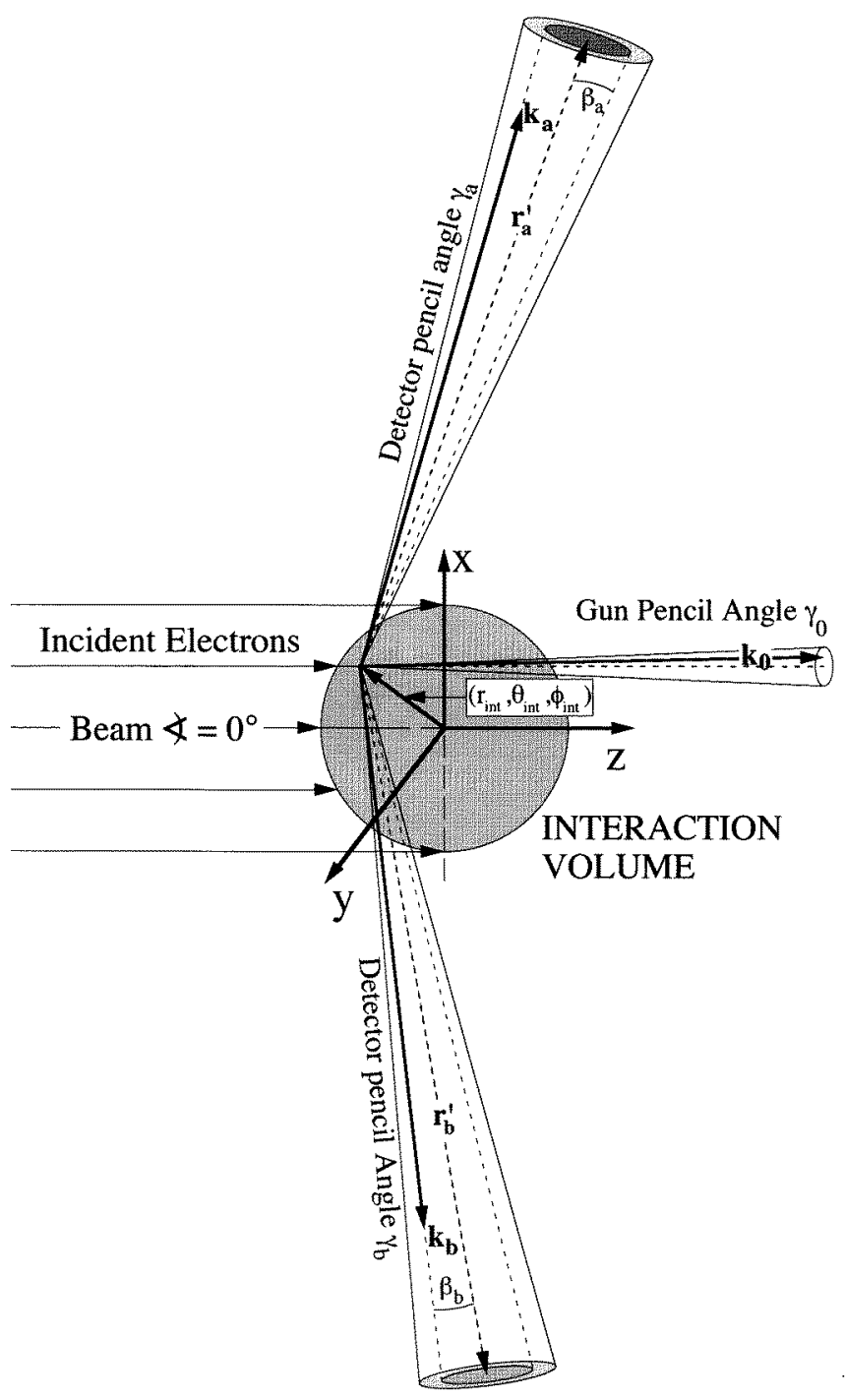

Figure 6. Details of the experimental geometry. The interaction region is defined by the overlap of incident electron beam and atomic beam. The incident electron beam has zero beam angle and a pencil angle, $\gamma_{0}$, defined by the apertures in the electron gun. The detectors view the interaction volume with pencil angles, $\gamma_{a}$, and $\gamma_{b}$, around the vectors $\boldsymbol{r}_{a}^{\prime}$ and $\boldsymbol{r}_{b}^{\prime}$, which are related to vectors from the centre of the interaction region $\boldsymbol{r}_{a}$ and $\boldsymbol{r}_{b}$ since $\boldsymbol{r}_{a, b}^{\prime}=\boldsymbol{r}_{a, b}-\boldsymbol{r}_{\text {int }}$. The experimental signal derives from summation over all coincidence events located at $\left(\boldsymbol{r}_{\mathrm{int}}, \theta_{\mathrm{int}}, \phi_{\text {int }}\right)$ in the interaction volume.

Since the angle, $\gamma_{a}$, is assumed to be small, this can be expanded as:

$$
A_{l_{a}}\left(\gamma_{a}\right)=1-\frac{l_{a}\left(l_{a}+1\right)}{8} \gamma_{a}^{2}+\cdots .
$$

Clearly an identical derivation is possible for the second analyser. Integration over the pencil angle, $\gamma_{0}$, of the incidental beam is simpler because there is not need to rotate the coordinate system, the attenuation again having the form given by equation (22). Hence the 
measured signal resulting from integration of the DCS at each point $\left(\boldsymbol{r}_{\mathrm{int}}, \theta_{\mathrm{int}}, \phi_{\mathrm{int}}\right)$ of the interaction region over the detection solid angles and incident electron beam pencil angle leads to an overall attenuation of each $I_{l_{a} l_{b} l_{0}}$ function as follows:

$$
I_{l_{a} l_{b} l_{0}}^{\text {ext }}\left(\theta_{a}, \phi_{a}, \theta_{b}, \phi_{b}\right) \rightarrow I_{l_{a} l_{b} l_{0}}\left(\theta_{a}, \phi_{a}, \theta_{b}, \phi_{b}\right) A_{l_{a}}\left(\gamma_{a}\right) A_{l_{b}}\left(\gamma_{b}\right) A_{l_{0}}\left(\gamma_{0}\right) .
$$

Since absolute experimental measurements are not conducted, the integration term over the event density profile $\rho\left(\boldsymbol{r}_{\text {int }}\right)$ simply results in an overall rescaling factor. Deconvolution of the experimental angular widths can therefore proceed using:

$$
B_{l_{a} l_{b} l_{0}}^{\text {Deconv }}=\frac{B_{l_{a} l_{b} l_{0}}}{A_{l_{a}}\left(\gamma_{a}\right) A_{l_{b}}\left(\gamma_{b}\right) A_{l_{0}}\left(\gamma_{0}\right)} .
$$

As an example, the attenuation of the $B_{776}$ parameter for the $(\mathrm{e}, 2 \mathrm{e})$ experiment at Manchester which has an input electron beam pencil angle of $2^{\circ}$ and detection cone angles of $3^{\circ}$ is 0.988 , a change of less than $2 \%$.

It should be noted that deconvolution must be carried out over all events that contribute to the coincidence yield, including those which result from asymmetric angles within the detector pencil cone angles and the pencil angle of the incident beam. The complete set of $B_{l_{a} l_{b} l_{0}}$ parameters must therefore be used, in contrast to the subset which has been presented here. These can be obtained by additionally measuring the cross section for asymmetric angles $\left(\theta_{a} \neq \theta_{b}, \phi_{a} \neq \pi-\phi_{b}\right)$ where the linear relationship of equation (10) no longer holds. Once these parameters are evaluated equation (25) can be used to deconvolve the cross section for both symmetric and asymmetric geometries. A small error therefore appears in Murray and Read (1993), since these authors did not consider all possible angles when attempting to deconvolve the DCS from the data. Rather only symmetric angles, $\left(\xi_{a}=\xi_{b}\right)$, were used and the effects of changes in the DCS with respect to the gun angle, $\psi$, were ignored.

\section{Summary and conclusions}

A comprehensive set of (e, 2e) experimental data for a helium target has been parametrized in a way that indicates the degree of correlation existing between ingoing and outgoing particles, and allows the number of partial waves contributing significantly to the reaction to be estimated. A least squares fitting method adopted to fit the data has been described, together with the results obtained from this fit.

The symmetric geometry chosen in the experiments reduces the number of contributing angular functions required to fit the data since many of the symmetric functions are related. Examples of these functions have been shown. The parametrization allows angular and energy dependencies of the cross section to be separated, and the energy terms have been further parametrized in terms of the excess energy shared equally between the outgoing electrons. This allows the DCS to be estimated for all angles at all energies from threshold to $50 \mathrm{eV}$ excess energy. Finally the parametrization is found to be advantageous when integrating the DCS over the angular acceptance angles of the analysers and the pencil angle of the incident electron beam. It has been shown that the form of the parametrization remains unchanged, only simple attenuation coefficients being necessary to deconvolve the experimental DCS and estimate the true DCS.

\section{Acknowledgments}

The UK Engineering and Physical Science Research Council is gratefully acknowledged for their financial support. We also acknowledge and appreciate useful discussions with Professor H Klar during the course of this work. 


\section{References}

Altick P L 1985 J. Phys. B: At. Mol. Phys. 181841

Berekdar J and Briggs J S 1994a J. Phys. B: At. Mol. Opt. Phys. 274271

-1994b Phys. Rev. Lett. 723799

Biedenharn L C 1960 Nuclear Spectroscopy part B (New York: Academic) 732

Biedenharn L C and Louck J D 1981 Angular Momentum in Quantum Physics (Reading, MA: Adison-Wesley)

Bowring N J, Murray A J and Read F H 1996 (submitted)

Brink D M and Satchler G R 1979 Angular Momentum (Oxford: Clarendon)

Bevington P R and Robinson D K 1990 Data Reduction and Error Analysis for the Physical Sciences (New York: McGraw-Hill)

Buckman S J, Gulley R J, Hoghbelahossein M and Bennett S J 1993 Meas. Sci. Technol. 41143

Coplan M A, Moore J H and Doering J P 1994 Rev. Mod. Phys. 66985

Crothers D S F 1986 J. Phys. B: At. Mol. Phys. 19463

Edmonds A R 1957 Angular Momentum in Quantum Mechanics (Princeton, NJ: Princeton University Press)

Fournier-Lagarde P, Mazeau J and Huetz A 1984 J. Phys. B: At. Mol. Phys. 17 L591

Hawley-Jones T J, Read F H, Cvejanovic S, Hammond P and King G C 1992 J. Phys. B: At. Mol. Opt. Phys. 25 2393

Klar H and Fehr M 1992 Z. Phys. D 23295

Maple V Reference Manual 1991 (London: Springer)

McCarthy I E and Weigold E 1995 Electron-Atom Collisions (Cambridge: Cambridge University Press)

Murray A J and Read F H 1992 Phys. Rev. Lett. 692912

-1993 Phys. Rev. A 473724

Murray A J, Read F H and Bowring N J 1994 Phys. Rev. A 49 R3162

Murray A J, Woolf M B J and Read F H 1992 J. Phys. B: At. Mol. Opt. Phys. 253021

Nelder J A and Mead R 1965 Comput. J. 7308

Peterkop R 1971 J. Phys. B: At. Mol. Phys. 4513

Pochat A, Zhang X, Whelen C T, Walters H R J, Tweed R J, Gélébart F, Cherid M and Allan R J 1993 Phys. Rev. A 47 R3483

Rau A R P 1971 Phys. Rev. A 4207

Röder J 1996 Private communications

Rose M E 1953 Phys. Rev. 91610

Rost J M 1995 J. Phys. B: At. Mol. Opt. Phys. 283003

Selles P, Huetz A and Maseau J 1987a J. Phys. B: At. Mol. Phys. 205195

Selles P, Mazeau J and Huetz A 1987b J. Phys. B: At. Mol. Phys. 205183 